\title{
Erratum to: Enforcing Memory Safety in Cyber-Physical Systems
}

\author{
Eyasu Getahun Chekole ${ }^{1,2(\bowtie)}$, John Henry Castellanos ${ }^{1}$, \\ Martín Ochoa ${ }^{1}$, and David K. Y. Yau ${ }^{1,2}$ \\ 1 Singapore University of Technology and Design, Singapore, Singapore \\ eyasu_chekole@mymail.sutd.edu.sg \\ 2 Advanced Digital Sciences Center, Singapore, Singapore
}

\section{Erratum to:}

Chapter "Enforcing Memory Safety in Cyber-Physical

Systems" in: S. K. Katsikas et al. (Eds.):

Computer Security, LNCS 10683,

https://doi.org/10.1007/978-3-319-72817-9_9

The original version of this chapter unfortunately contained a mistake. The first author's name was incorrect. The correct name should read: Eyasu Getahun Chekole.

The updated online version of this chapter can be found at https://doi.org/10.1007/978-3-319-72817-9_9 